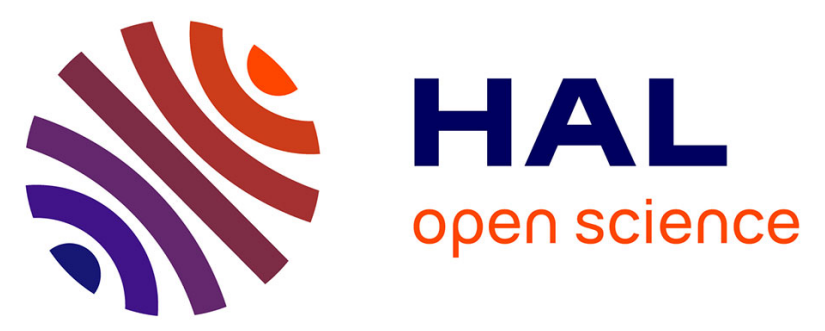

\title{
Dual Stage Options for Interface Designs Suitable for Haptic Interaction at the Micro-Nano Scales
}

Abdenbi Mohand-Ousaid, Tianming Lu, Cécile Pacoret, Stéphane Régnier, Vincent Hayward

\section{To cite this version:}

Abdenbi Mohand-Ousaid, Tianming Lu, Cécile Pacoret, Stéphane Régnier, Vincent Hayward. Dual Stage Options for Interface Designs Suitable for Haptic Interaction at the Micro-Nano Scales. 2014 International Symposium on Experimental Robotics, Jun 2014, Marrakech, Morocco. hal-01419726

\section{HAL Id: hal-01419726 \\ https://hal.sorbonne-universite.fr/hal-01419726}

Submitted on 19 Dec 2016

HAL is a multi-disciplinary open access archive for the deposit and dissemination of scientific research documents, whether they are published or not. The documents may come from teaching and research institutions in France or abroad, or from public or private research centers.
L'archive ouverte pluridisciplinaire HAL, est destinée au dépôt et à la diffusion de documents scientifiques de niveau recherche, publiés ou non, émanant des établissements d'enseignement et de recherche français ou étrangers, des laboratoires publics ou privés. 


\title{
Dual Stage Options for Interface Designs Suitable for Haptic Interaction at the Micro-Nano Scales
}

\author{
Abdenbi Mohand Ousaid ${ }^{\star}$, Tianming $\mathrm{Lu}^{\star}$, Cécile Pacoret, \\ Stéphane Régnier, and Vincent Hayward \\ Sorbonne Universités, UPMC Univ Paris 06, \\ UMR 7222, ISIR, F-75005, Paris, France \\ \{mohand_ousaid, lu, pacoret\}@isir.upmc.fr \\ \{regnier, hayward\}@upmc.fr \\ http://www.isir.upmc.fr
}

\begin{abstract}
Direct, manual interaction with the micro/nano scales is not straightforward because the objects at this scale obey unituitive physics. For instance, in ambient conditions at the micro-scale, capillary forces dominate over many other forces. When the scale becomes smaller, Brownian motion becomes pervasive. Haptic interfaces give us the option to bring the experience of this physics with the direct reach of the human sensorimotor capabilities. To cope with the limitations of conventional force feedback devices, we present here two alternative dual-stage designs suitable to address the needs of the interaction with the micro/nano scales. The first one features very low apparent inertia, a large dynamics range and a wide bandwidth. This properties are obtained by coupling a large actuator to a small one via a viscous coupler. Feedback can then be used to achieve nearly perfect transparency. The second is a conventional force feedback device augmented with a tactile transducer. The two channels are frequency compensated to achieved a flat response fro DC to one $\mathrm{kHz}$.
\end{abstract}

Keywords: Haptic interface, Dual-stage architecture, Pantograph, Transparency, Tactile transducer, Signal crossover.

\section{Introduction}

At the nano and micro scales, the mechanical behavior of objects no longer is dominated by gravity and friction. At short range, forces including electrostatic, capillary, and van der Waals forces are preeminent [1]. As a result, the physics of the micro/nano scales differs completely from that of the macro scale and are not accessible to humans sensory and motor capabilities.

Like a microscope magnifies viewed objects, haptic devices can be employed to magnify interaction between objects at a scale at which humans are naturally

\footnotetext{
^ Contributed equally to this work.
} 
effective. Most haptic devices described in the past fail to match the human sensorimotor capacity and thus act as an obstacle between the hand and the phenomenon that could be accessed. Conventional interfaces are subjected to inherent friction and/or high inertia that affect the interface transparency, dynamic range, and bandwidth.

At the micro/nano scales, three issues arise in term of interface design [2]. Effective scaling systems must provide high transparency to convey the most of what can be sensed by humans and not taint it with noise; they must have a large dynamic range since human can motorically and sensorially deal with 4-5 orders of magnitude; and they must have wide bandwidth since the haptic capabilities cover from dc to about $1 \mathrm{kHz}$. Transparency thus quantifies a haptic interface's capacity to reproduce micro/nano interactions to a human operator. The dynamic range is related to the force resolution of the interface. It quantifies the ratio of the largest to the smallest forces that can be commanded, and thus sensed, through the interface. Bandwidth refers to the capacity of the interface to convey mechanical signals over a large frequency range, in order to render complex phenomena, such as Brownian motion.

In the past 25 years, several haptic interfaces have been proposed to deal with interaction with small objects. They enhance human interaction capabilities at the microscale using either electrodynamic transducers $[3,4]$, or single stage systems based on conventional robotic architectures, see [5], among several other examples. Such interfaces are subjected to inherent friction and high inertia that contribute spurious forces that mask the mechanical signals to be felt by the users. With single stage systems, sacrifices must be made regarding transparency, dynamic range, or the bandwidth in favor of other factors such as maximum force. To cope with these limitations, we describe here two alternative dual-stage haptic device designs (see Fig. 1) suitable to address the needs of interactions at the micro/nano scales.
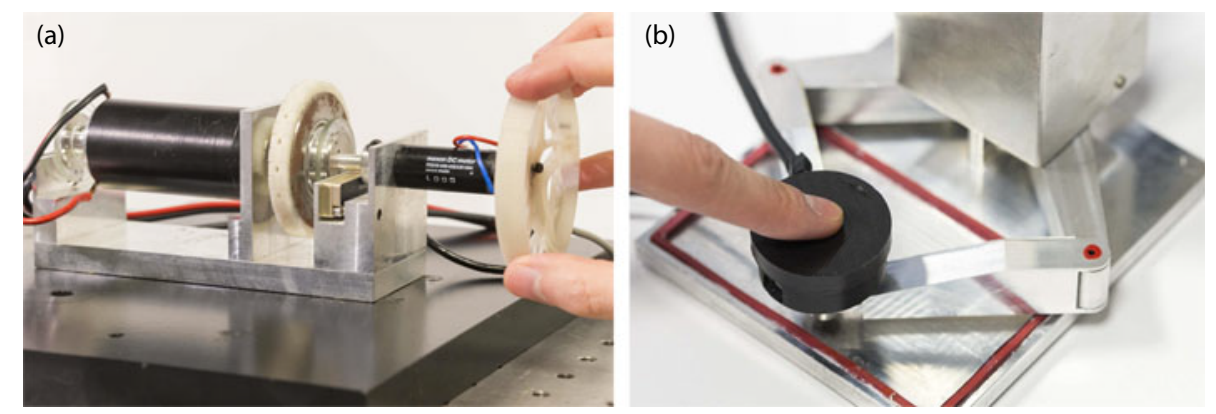

Fig. 1. (a) Dual-stage haptic interface with viscous coupling, (b) Direct drive haptic interface augmented with high-frequency tactile transducer. 


\section{Viscously coupled dual stage design}

The first design, see Fig. 1(a), focuses on transparency and dynamic range. It is based on a dual-stage architecture [6,7]. The large, proximal motor provides power and the small, distal motor reproduces the force transients. The two stages are connected to each other by a passive viscous coupler based on Foucault (eddy) currents. Such coupler transforms slip velocity into torque with exacting accuracy and exhibits a linear behavior over a large velocity range. The viscous torque is proportional to the relative velocity between proximal and distal motor, $\tau_{\text {coupler }}=b\left(\dot{\theta}_{1}-\dot{\theta}_{2}\right)$. The handle is driven by the sum of two torques, that of the coupler and that of the distal motor. A fundamental advantage is the possibility to decouple the output torque from the inertia of the motor. Referring to Fig. 2, a pole-placement controller, $C(z)$, designed by polynomial methods was shown to be able to achieve a near perfect transparency [7].

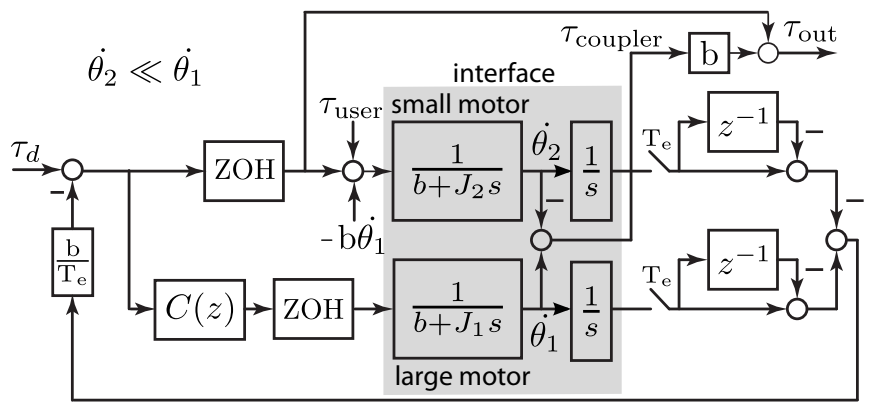

Fig. 2. The reference torque, $\tau_{d}$, is compared to the torque produced by the coupler, $\tau_{\text {coupler }}$. The large motor is enslaved by the compensator $C(z)$ to zero the torque error. The small motor compensates for the slower response of the large motor.

Figure 3(a) shows the response to a null reference torque. The torque felt by the operator, $\tau_{\text {out }}$, is nearly zero despite rapid movements of the handle. The inertia of the proximal motor is entirely masked by the feedback control. Mechanically speaking, the power required to move the large motor is entirely supplied by the power amplifier and not by the operator. Only the inertia of the small motor is felt (Fig 3b).
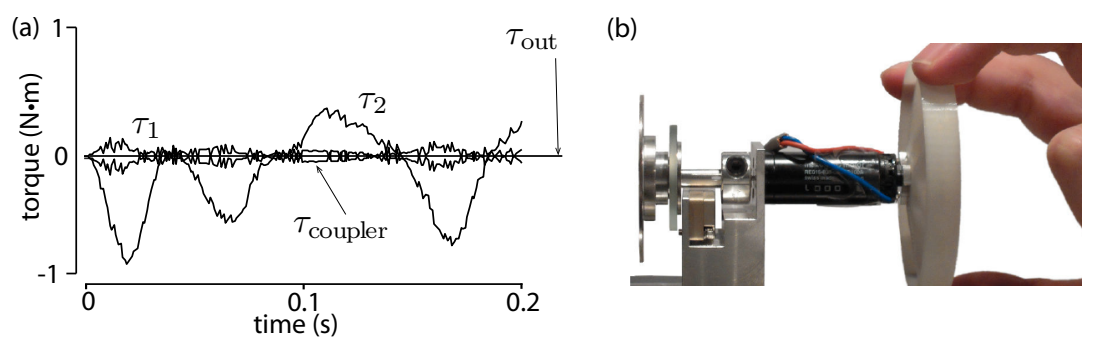

Fig. 3. (a) Interface response to a null reference. (b) What is felt by the user. 


\subsection{Interface evaluation}

The objective was to verify that the high-level degree of transparency of the dual-stage had a measurable effect on human performance when using the interface. Human observers to performed a signal detection task using the stimulus depicted Fig. 4(a). One component had a fixed magnitude and the other varied from imperceptible values to easily detectable values. To create an experimental condition that resembled actual use, observers detected the presence of weak force perturbations at random locations in the workspace. The stimuli was felt through two identical handles, one connected to the large motor, and other to the small motor, see Fig. 4(b).

(a)

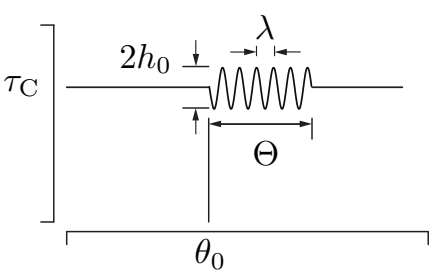

(b)

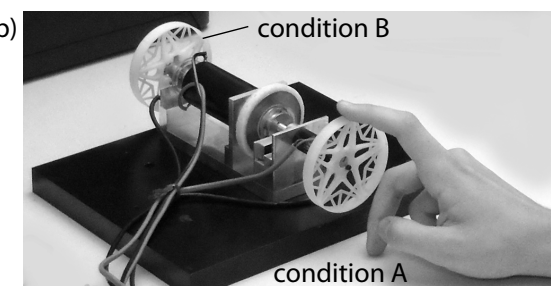

Fig. 4. Dual-stage interface validation. (a) In condition $A$, the handle was connected to the output of the dual-stage drive. In condition $\mathrm{B}$, an identical handle was connected directly to the shaft of the proximal motor and the coupler was disconnected. (b) Stimulus. A small burst of oscillation of size $\Theta$, amplitude $h_{0}$, and spatial period $\lambda$, was superposed onto a larger friction force, $\tau_{\mathrm{C}}$.

The observers were not aware of the condition under which they were working, that is with the dual-stage interface (condition A) or with the single motor (condition B), similar to a conventional interface. All subjects produced typical psychometric curves as shown in Fig 5. Despite inter-subject variations, it could be concluded that the performance detection of the observers was improved by almost an order of magnitude. As a whole, the interface achieves a large dynamic range which goes from $5.2 \mathrm{mN}$ to $5.7 \mathrm{~N}$ that is three orders of magnitude. Reader can find in [7] more details about the evaluation procedure.
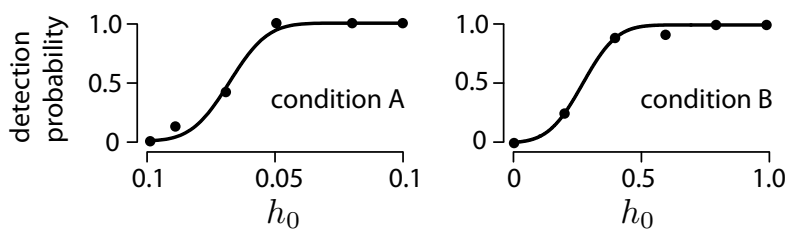

Fig. 5. Results and fits for conditions A and B a typical observer. 


\subsection{Haptic interaction with a water droplet}

To test the ability of the haptic interface to bring the physics of micro scales to a human scale perception, we experimented with a actual interaction at micro scales. The test was carried out on a complex case for microscales force sensing, which entails measuring and feeling the interaction of a thin glass probe with a water droplet. The droplet was first approached towards the probe using the position of the interface handle as a set point for the droplet micro-positioner. Then, the droplet was contacted and retracted in approach-retraction cycle. The interaction force was measured by using a micro force sensor $[8,9]$ and fed back to the user through the dual-stage haptic interface. The dual-stage interface conveys the amplified interaction forces between the glass probe and water droplet at a scale where the operator could interact through his/her natural touch perception. Figures 6(a) and 6(b) represent respectively the force measured by the sensor over a cycle of approach-retraction and the force transmitted to the operator by a probe with a diameter of $80 \mu \mathrm{m}$. The force felt by the user through the interface handle was exactly the force measured by the sensor amplified by a coefficient $\alpha_{f}=0.0510^{6}$. his system shows the capacity of the haptic interface to let humans to go beyond their sensory limitations. The system was also tested by several users with no experience in microscale phenomena. Various interaction forces, like pull-in and pull-off forces, are correctly rendered to the operators. Readers can refer to [10] for more details about the set-up and the experiment.

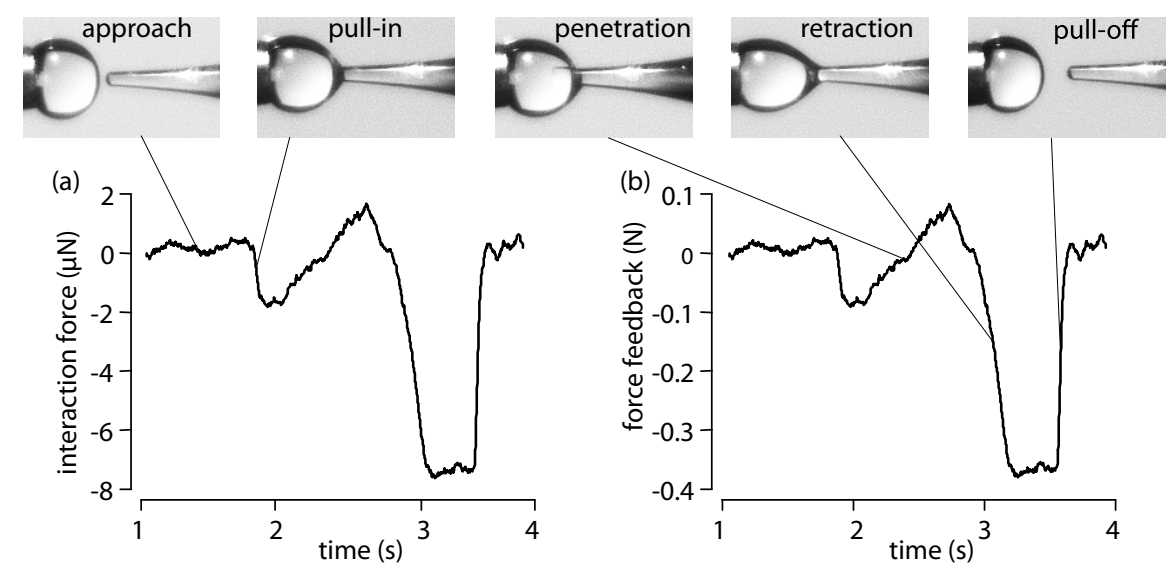

Fig. 6. Illustration of the main phases during the interaction, approach, pull-in, penetration, retraction and pull-off. Measuring and feeling the interaction between a glass probe and water droplet. (a) Interaction force measured by the sensor over a cycle of approach-retraction according to time. (b) Haptic force feedback felt by the operator through the dual-stage haptic interface. . 


\subsection{Discussion}

We described a new type of electromagnetic drive for use in haptic interfaces and other applications. The interface increases its dynamic range and its transparency by decoupling the delivered maximum torque from its effective inertia. As a whole, the interface achieves a large dynamic range which goes from $5.2 \mathrm{mN}$ to $5.7 \mathrm{~N}$. In terms of transparency for a human user, the interface achieves two orders of magnitude of improvement over existing designs. However, the capacity of the haptic interface to convey large frequency signals remains limited, particularly tactile sensations that can be employed to feel fast phenomana like Brownian motion. To address this need, augmenting a conventional force feedback device with a vibrotactile transducer is an interesting option. Such as approach is described in the next section.

\section{Haptic interface augmented with tactile transducer}

A significant challenge is to increase the bandwidth of an interface to enable key aspects of the rich information available in the micro/nano scales to be felt $[1$, 11-13]. The problem arises from factors such as the presence of resonances in the mechanical structure of articulated systems of a scale sufficient to interact with a moving hand. Because of these resonances, typical haptic interfaces must be limited in bandwidth using appropriate low-pass filters [14]. To fix ideas, the best performing Sensable Phantom 1.0A device has a useful mechanical bandwidth of only $30 \mathrm{~Hz}$. As a result, high frequency phenomena such as Brownian motion, van der Waals and electrostatic forces cannot be reproduced by the interface. The mechanics of cantilevered structures of appropriate scales preclude any device to achieved a bandwidth able to transmit the signals arising from the micro/nano scales. In order to address this problem, we constructed a dual-stage design based on channel separation using signal crossover to separate haptic signals into low and high frequency channels that recombine at the tip of the device.

The main stage was a 'Pantograph' haptic interface, which is a planar parallel mechanism of two actuated degrees of freedom. It provided a stiff and light set of linkages driven without transmission $[15,16]$. It has the desirable features of light inertia, high stiffness, compact design but large operating workspace. The mechanical properties give rise to uniform magnitude performance on the workspace. A vibrotactile transducer, called a 'Haptuator' [17], was embedded inside the finger interface to reproduce fast oscillations. The Haptuator, based on the principle of conservation of momentum, is designed to work in the high frequency regions and is limited in the low frequency range because of displacement saturation. The resulting system can be viewed in Fig. 1(b). Each component operated in its optimal range of frequencies. From the knowledge of the identified system's dynamics, a compensation/crossover scheme could achieve nearly ideal, uniform magnitude performance over the whole frequency band, from DC up to $1.0 \mathrm{kHz}$ and up. The overall system block diagram is shown in Fig. 7 . 


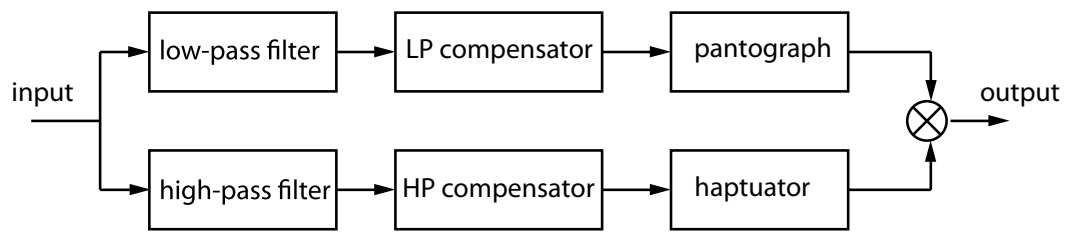

Fig. 7. System block diagram with compensation and crossover, L-P and H-P stand for low-pass and high-pass respectively.

\subsection{Interface evaluation}

The magnitude performance of each subsystem was first experimentally determined using identification by the third of octave band method. Then, the compensation scheme and the crossover filter were implemented in a real-time computational system. The magnitude response over the required frequency bands, from DC to $1.0 \mathrm{kHz}$, is reported in Fig. 8. It can be seen that the interface exhibited a flat response in the desired frequency region.

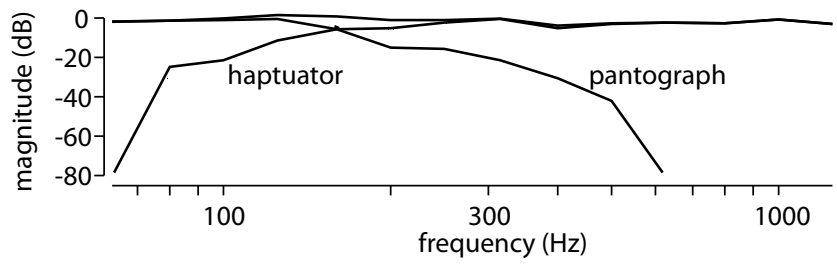

Fig. 8. Acceleration magnitude response of the interface with tactile feedback. The result shows a flat response up to $1.0 \mathrm{kHz}$.

\subsection{Optical tweezers application}

The interface was employed in a simulation scenario for an optical tweezers application to demonstrate its capabilities [18, 19], see Fig. 9(a). The scenario involved a nano-scale spherical object placed in a liquid environment which was subjected to nothing else but the Brownian motion. Its movements were recorded but a novel high-speed image capture system [18]. During operation, operators were able to feel high frequency oscillations. In order to verify that the interface was capable of rendering the Brownian motion sensed by the optical tweezers simulation, a histogram was plotted to show the distribution of the signals recorded by an accelerometer mounted on the finger interface, see Fig. 9(b). It can be concluded that the measurements reflected the expected normal distribution and that the haptic interface was capable of rendering Brownian motion. Observers invited to test the interface reported that the haptic sensations where novel and provided new intuition about what Brownian motion is. 

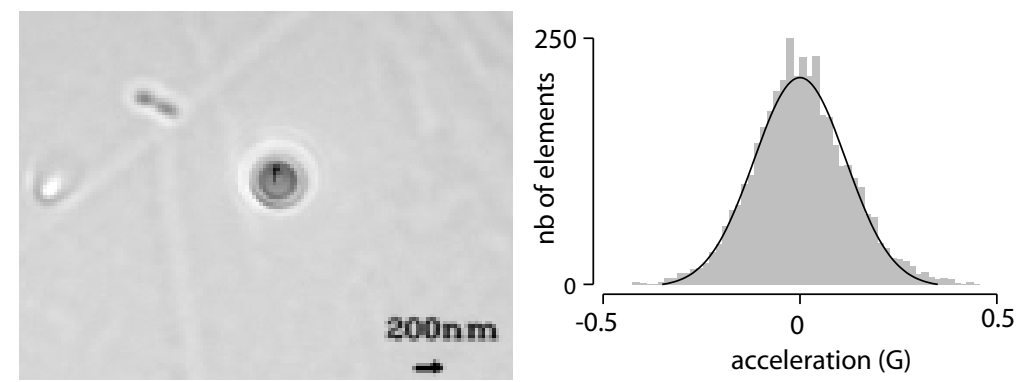

Fig. 9. (a) A virtual scenario created from records of an optical tweezers. The $200 \mathrm{~nm}$ spherical object was placed in a liquid environment and thus was subjected to Brownian motion. (b) Histogram of measured acceleration had a quasi-normal distribution. The results suggest the measurements conformed to a Gaussian distribution and the interface was able to render Brownian motion.

\subsection{Discussion}

A haptic teleoperation interface was developed by combining a Pantograph and an Haptuator, which has uniform performance over a large frequency band from DC to $1.0 \mathrm{kHz}$. By applying the interface to data collected from optical tweezers, the observers were able to experience the Brownian motion present in the micro/nano world. The presented system demonstrates high bandwidth capability and reliability over determined frequency band. It gives rise to potential possibilities for complex applications which require uniform performance over large frequency regions. Further research aims at combining this concept with the viscously coupled dual stage design so that wide bandwidth, high transparency and dynamic range could be achieved all in one single system.

\section{Conclusion}

Dual-stage architectures were proposed to overcome the performance limitations of conventional haptic interfaces. A knob-type high fidelity haptic interface with one degree of freedom based on viscous coupling is advantageous when high torques are required since inertia of large motors are decoupled from operator experience. A dual stage design based on signal crossover and compensation filters could extend conventional interface bandwidth considerably. In future works, the complementary between the first and second design could be exploited to design high-performance and compact haptic interfaces suitable for dealing with micro/nano scales interactions.

\section{Acknowledgments}

This work was supported in part by the eurostar project REMIQUA. 


\section{References}

1. M. Gauthier and S. Régnier. Robotic microassembly, Wiley-IEEE press, publisher, 2010.

2. V. Hayward and O. R. Astley. Performance measures for haptic interfaces. In G. Giralt and G. Hirzinger, editors, Robotics Research: The 7th International Symposium, pages 195-207, Heidelberg, 1996. Springer Verlag.

3. R. Hollis, S. Salcudean, and D. Abraham. Toward a tele-nanorobotic manipulation system with atomic scale force feedback and motion resolution. Micro Electro Mechanical Systems conference, pp. 115-119, 1990.

4. I. W. Hunter, S. Lafontaine, P. M. F. Nielsen, P. J. Hunter, and J. M. Hollerbach. Manipulation and dynamic mechanical testing of microscopic objects using a telemicro-robot system. IEEE Control Systems Magazine, vol. 10, nº 2, pp. 3-9, 1990.

5. I. Gaponov, J-H. Ryu, S.-J. Choi, H.-C. Cho, and Y. Poduraev. Telerobotic system for cell manipulation. IEEE/ASME International Conference on Advanced Intelligent Mechatronics, pp. 165-169, 2008.

6. G. Millet, S. Haliyo, S. Rǵnier, and V. Hayward. The ultimate haptic device: First step. Eurohaptics Conference and Symposium on Haptic Interfaces for Virtual Environment and Teleoperator Systems. World Haptics, 2009, pp. 273-278.

7. A. Mohand-Ousaid, G. Millet, S. Régnier, S. Haliyo, and V. Hayward. Haptic interface transparency achieved through viscous coupling. International Journal of Robotics research, vol. 31, $\mathrm{n}^{\circ} 3$, pp. 319-329, 2011.

8. A. Mohand Ousaid, S. Haliyo, S. Régnier and V. Hayward. Active electrostatic force-sensing probe in the microNewton range. IEEE/ASME International Conference on Advanced Intelligent Mechatronics, pp. 612-617, 2013.

9. A. Mohand Ousaid, S. Haliyo, S. Régnier and V. Hayward. H-infinity optimal control enabled micro-force sensing. 3d International Conference on Systems and Control, pp. 490-495, 2013.

10. A. Mohand Ousaid, A. Bolopion, S. Haliyo, S. Régnier and V. Hayward. Stability and transparency analysis of a teleoperation chain for microscale interaction. IEEE International Conference on Robotics and Automation, 2014, in press.

11. J.M. Romano and K.J. Kuchenbecker. Creating realistic virtual textures from contact acceleration data. IEEE Transactions on haptics, vol 5, $\mathrm{N}^{\circ} 2$, pp. 109-119, 2012 .

12. M. Sitti. Micro and nano-scale robotics. American Control Conference, vol. 1, pp. $1-8,2004$.

13. B. Finio, K. Galloway and R. Wood. An ultra-high precision, high bandwidth torque sensor for microrobotics applications. IEEE/RSJ International Conference on Intelligent Robots and Systems, pp. 31-38, 2011.

14. G. Campion and V. Hayward. Fundamental limits in the rendering of virtual haptic textures. Eurohaptics Conference and Symposium on Haptic Interfaces for Virtual Environment and Teleoperator Systems, pp. 263-270, 2005.

15. Hayward, V., Choksi, J. Lanvin, G., and Ramstein, C. Design And Multi-objective optimization of a linkage for a haptic interface. In Advances in Robot Kinematics. J. Lenarcic and B. Ravani (Eds.). Kluver Academic. pp. 352-359, 1994.

16. Campion, G., Wang, Q., and Hayward, V. The pantograph Mk-II: A Haptic instrument. IEEE/RSJ International Conference on Intelligent Robots and Systems, pp. 723-728, 2005.

17. H.-Y. Yao and V. Hayward. Design and analysis of a recoil-type vibrotactile transducer. The Journal of the Acoustical Society of America, vol. 128, nº 2, pp. 619-627, 2010. [Online]. Available: http://link.aip.org/link/?JAS/128/619/1 
18. Z. Ni, C. Pacoret, R. Benosman, and S. Régnier. 2d high speed force feedback teleoperation of optical tweezers. IEEE International Conference on Robotics and Automation, pp. 1700-1705, 2013.

19. Z. Ni, C. Pacoret, R. Benosman, S. Ieng, and S. Régnier. Asynchronous event based high speed vision for micro-particles tracking. Journal of microscopy, vol. 245, $\mathrm{n}^{\circ}$ 3, pp.236-244. 2011. 